\title{
Skull base metastases from a malignant solitary fibrous tumor of the liver. A case report and literature review
}

\author{
Lu Peng, Yang Liu, Yongbiao Ai, Zhisu Liu, Yueming He and Quanyan Liu*
}

\begin{abstract}
Solitary fibrous tumors (SFTs) of the liver are rarely described; only 38 cases have been reported in literature, most of which have shown benign clinical characteristics, and only 3 of these cases exhibited malignant variants. In this study, we present a 24-year-old woman with a 1-month history of a rapidly enlarging abdominal mass and a CT showing an exophytic heterogeneous liver mass with a firm parietal bone mass. The patient underwent a transcatheter arterial chemoembolization (TACE) before operation, and an extended right hepatectomy and craniectomy with a negative margin was performed under general anesthesia. The masses showed histological features of oval spindle cells haphazardly arranged in the classic short-storiform or so-called patternless pattern of solitary fibrous tumors. The tumor cells showed positive immunohistochemical reactions to CD34 and bcl-2. The tumor recurred in the residual liver 2 months after operation, metastatic osteoblastic lesions in the thoracic and lumbar vertebrae were identified 3 months after the operation, and lumbar vertebrae metastasis 7 months after operation paralyzed the patient. The patient underwent percutaneous ethanol injection therapy (PEI) and chemotherapy, but the patient died because of the uncontrolled tumor 16 months after the initial operation. To our knowledge, this is the first case of malignant solitary fibrous liver tumors with skeletal metastasis.
\end{abstract}

Keywords: Solitary fibrous tumor, Liver

\section{Introduction}

Solitary fibrous tumors (SFTs) are a rare soft-tissue neoplasm that was initially described most commonly in the pleural cavity[1]. In the past decade, SFTs have been discovered at many body sites, including the liver (mostly the right lobe), orbits, superior respiratory tract, abdomen, breast and soft tissue [2-4]. SFTs are spindle cell neoplasms. The histogenesis of SFT has been a matter of contention and still has not been definitively discerned, but a mesenchymal origin is favored over a mesothelial origin[5].

More than $80 \%$ of SFTs are benign, asymptomatic and slow-growing tumors, but malignant and symptomatic forms, most often in the pleura, have been described $[6,7]$. Clinical and radiological findings are not specific and cannot exclude malignancy. Preoperative cytology may be inconclusive or misleading. Notably, all SFTs

\footnotetext{
* Correspondence: spss2005@126.com

Department of General Surgery, Research Center of Digestive Diseases, ZhongNan Hospital, Wuhan University, Wuhan 430071, P.R. China
}

that recurred or metastasized after resection in some larger series were $\geq 10 \mathrm{~cm}[8,9]$. In addition, several authors describe a histologically malignant component with increased cellularity and mitoses in otherwise benign-appearing SFTs, as a frequent occurrence in tumors that recurred or metastasized [8-10]. The recommended treatment of SFT is surgical resection. Despite this treatment, SFTs can recur and metastasize and have reported 10-year overall survival rates of 54$89 \%$ after a complete surgical resection [11].

However, surgical experience with SFTs of the liver is notably limited. In one series, $13.5 \%$ of 37 pleural SFTs metastasized [12]. SFT is rarely located in the liver parenchyma and usually follows a benign course. Few case reports in the literature describe a local recurrence of liver SFT and even fewer describe metastases [12-16]. In this study, we report a case of malignant liver SFT with skeletal metastasis and discuss the clinical presentation, previous diagnoses, radiological characteristics, histopathological and immunohistiochemical features, 
surgical treatment, adjunctive therapy and prognosis of SFTs with a review of the literature.

\section{Case report}

A 24-year-old, unmarried, nulliparous woman had presented one month earlier with upper right abdominal discomfort, distention, and vague right quadrant pain. The patient was a shop assistant for 4 years with no known history of potential environmental hazardous substances exposure. In addition, she was also a nonsmoker. Except for being a carrier of hepatitis B, she had no relevant medical or family history. For the further evaluation of her symptoms, she was referred to the referral hospital, where a contrast enhanced computer tomography (CT) study revealed a 30-cm exophytic heterogeneous mass in the right lobe of the liver (Figure 1 ). In a 2-D view, the lesion appeared as a hypodense area without calcifications measuring $28 \mathrm{~cm} \times 18 \mathrm{~cm}$. An arterial-phase infusion of contrast showed peripheral enhancements with thin linear areas of enhancement (probably vessel-related) in the lesion. In the portal phase, there was a dishomogeneous enhancement with multiple hypodense areas. In the late phase, the lesion showed a light enhancement (similar to normal liver tissue) with multiple strong hypodense areas. There was no presence of ascites, and the patient had been given hepatinica. The patient was admitted to our hospital for surgery.
On examination, abdominal distension was present. Palpation revealed tenderness and a large, firm mass in the right hypochondrium and epigastrium, and the liver was $12 \mathrm{~cm}$ below the lower edge of the ribs. There was no evidence of ascites, edema, hepatic failure, or cirrhosis stigmata. A firm mass of $4 \mathrm{~cm} \times 5 \mathrm{~cm} \times 3 \mathrm{~cm}$ was present on the right parietal bone without any tenderness (Figure 2). Laboratory tests, including routine biochemistry, liver function tests, urinalysis were normal, and tumor markers, such as serum carbohydrate antigen (CA)-125 was abnormal, whereas cancer antigen (CEA), $\alpha$-fetoprotein, and CA19-9 levels were in the normal ranges. The patient's cranial CT scan revealed a parietal mass that was thought to be a metastatic tumor. On a whole-body skeletal scan, increased activity was found in the right parietal bone.

The patient underwent angiography and transcatheter arterial chemoembolization (TACE, using diamminedichloroplatinum $60 \mathrm{mg}$, 5-fluorodeoxyuridine $1000 \mathrm{mg}$, Epirubicin $30 \mathrm{mg}+18-\mathrm{ml}$ Lipiodol emulsifier) of the tumor-supplying vessels a few days before operation (Figure 3). A laparotomy was performed 8 days after admission and revealed that the tumor had pushed the colon to left and had adhered to the colon, stomach and omenta. An extended right hepatectomy was performed, and the postoperative course was uneventful. The gross examination revealed a large tumor measuring $30 \times 17$ $\times 15 \mathrm{~cm}$ and weighing $3750 \mathrm{~g}$ that was a firm, whitish,
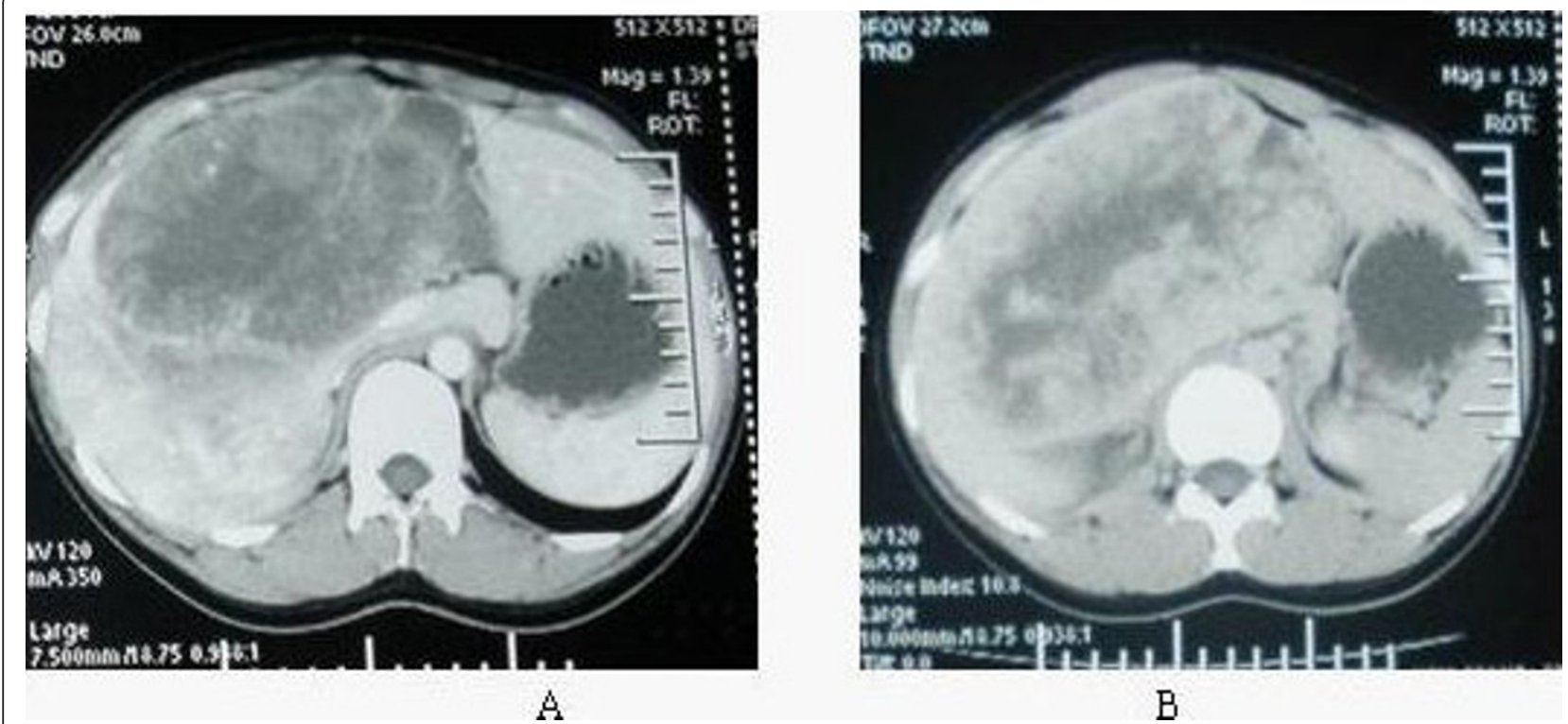

Figure 1 Enhanced CT scan before hepatectomy. A: Arterial phase image showing a large, heterogeneous mass of the liver with hypodense areas, which had clear border between tumor and the normal liver. Lesion showed heterogeneous enhancement and the high density of tumor blood vessels was seen in this region, lower density area to the central region as the center radial arrangement. Left hepatic vein was be compressed by the tumour and turned out to be rigidity. B: Venous late phase images showing a large, solid mass of the liver. Large hypodense tumor of the right hepatic lobe with an enhancement of the peripheral portion. The tumour was diffuse enhanced with a lager hypodense area in the center thought to be necrosis. 


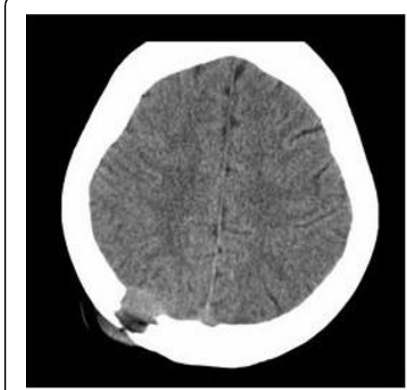

A

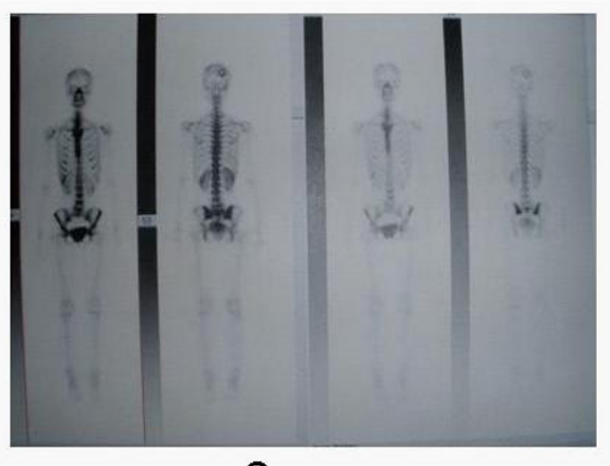

C

Figure 2 Metastatic tumor of the right parietal bone. A: CT scan of the brain shows that the right parietal bone is ruined before the craniectomy. B: CT scan of the brain after craniectomy. C: The emission-computed tomography scan shows an abnormal distribution of radioactivity in the right parietal bone area; the sparse distribution of radioactivity was central; peripheral distribution of radioactivity uptake before craniectomy.

fibrotic nodule-encapsulated mass with necrosis (Figure 4). The resection margins were tumor-free. The tumor was located in the liver parenchyma, not in relation to the serosal surface. The cells were spindle-shaped tumor cells that were highly cellular and pleomorphic with areas of necrosis and a high mitotic index (>10/hpf), haphazardly arranged in the classic short-storiform or so-called patternless pattern, and invading the surrounding liver tissue (Figure 5). The immunohistochemical analysis was strong positive for CD34, Bcl-2 and Vimentin. Ki-67 antigen staining was 3-5\% positive. The tumor cells were negative for other markers, such as CD117, SM-actin, S-100, CD68, Myo-D1, CD99, Desmin, CD31, Calretinin, Synaptophysin, Cytokeratin, and Keratin (Figure 6). A craniectomy was performed 13 days after the extended right hepatectomy. The pathological examination showed skull base metastases from a malignant solitary fibrous tumor of the liver.

A postoperative MRI examination in July 2010 demonstrated that the postsurgical residual lesion was still large. At follow-up one month later, the patient had severe back and waist pain. An MRI identified metastatic osteoblastic lesions of the thoracic and lumbar vertebrae (Figure 7).

The patient underwent four percutaneous ethanolinjection therapies (PEIs) and was treated with four rounds of chemotherapy that included adriamycin ( 40 $\mathrm{mg}$ on days 1-3), ifosfamide (3.0 g on days $1-4)$, and mesna ( $0.6 \mathrm{~g}$ IV $0 \mathrm{~h}, 4 \mathrm{~h}$, and $8 \mathrm{~h}$ after IFO). Adjunctive radiation therapy was not administered. Despite the treatment, the tumor relapsed rapidly and was markedly enlarged. At follow-up seven months later, the patient was paralyzed; the patient died 16 months after the initial operation.

\section{Discussion}

Hepatic SFTs are unusual neoplasms, with 38 previous cases worldwide being reported in the literature. SFTs typically affect adults aged 54.6 years (16-83) with no underlying predisposing factors. In a review article of 14 published cases, a strong female predilection was noted, and the average follow-up period was 27 months

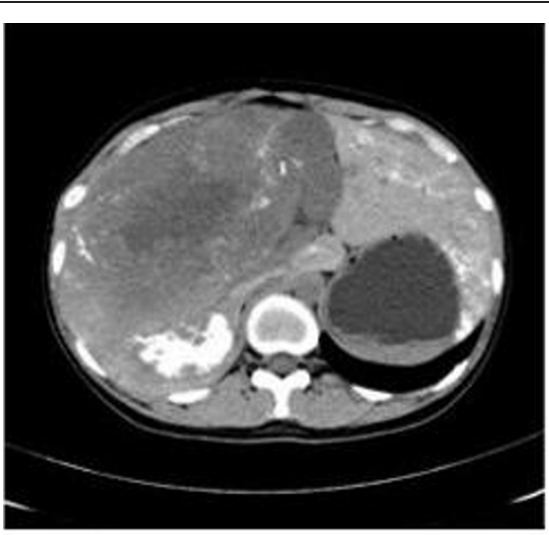

A

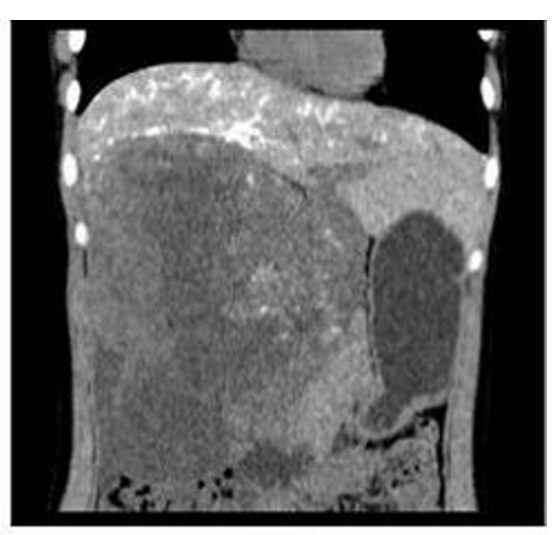

B

Figure 3 CT scan after the treatment of TACE shows that there is almost no lipiodol deposits in the tumor area. 


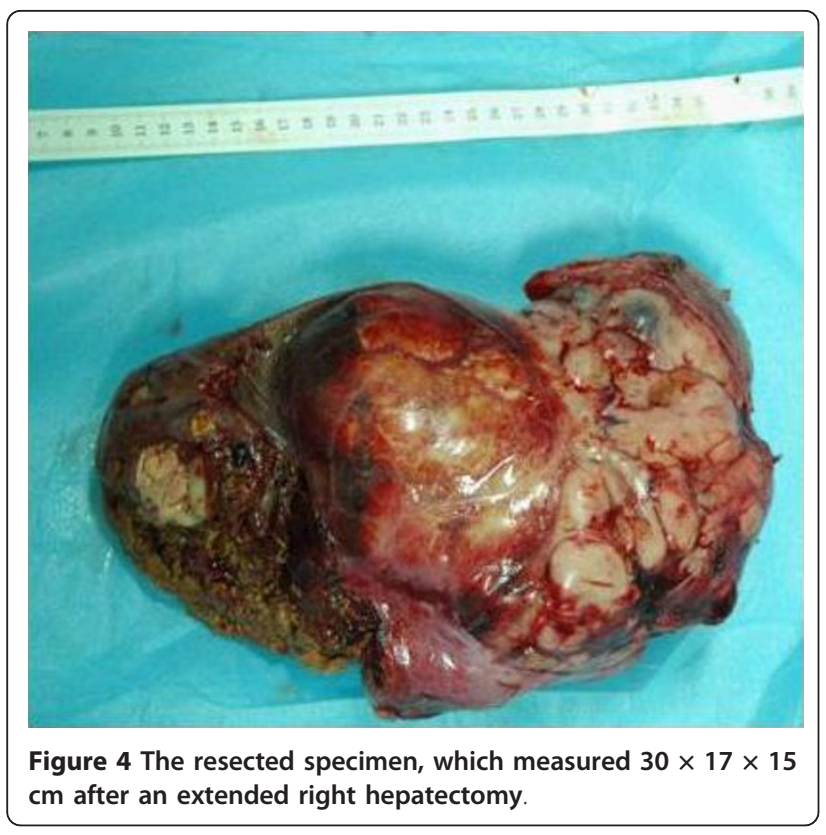

$[17,18]$. Most of these cases showed benign clinical characteristics, and only 3 cases had malignant variants. The clinicopathological features of the malignant hepatic SFT cases reported are summarized in Table 1. Three well-described cases, including the present case, came from one woman and two men with SFTs in the right lobe of the liver, and these patients ranged in age from 25 to 70 years (mean: 49). Immunohistochemically, the tumor cells showed strong vimentin immunostaining in all three cases and were positive for CD34 in two cases but negative for EMA and S-100 protein. The present case seems to be the first case of a hepatic malignant solitary fibrous tumor with skeletal metastases.
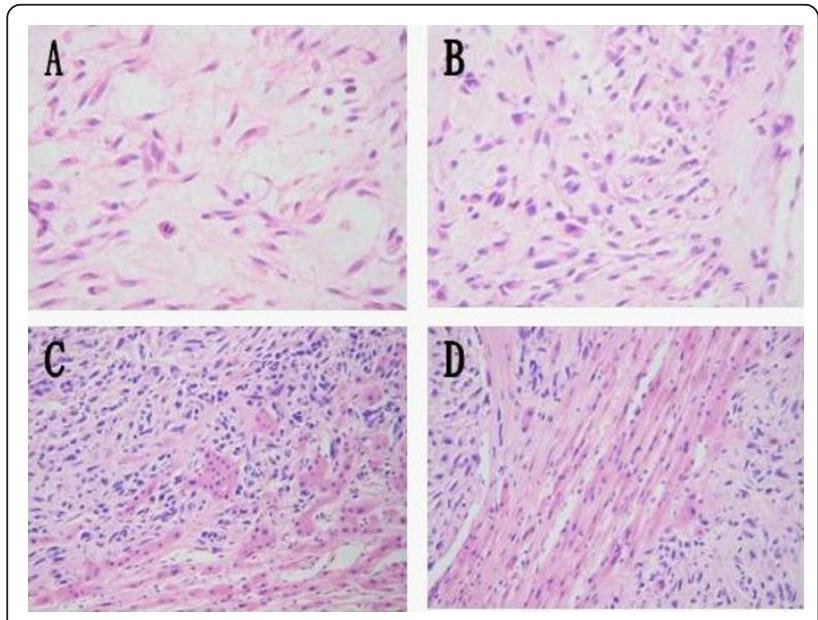

Figure 5 Microscopic appearance. (A) The mitosis of the nucleus. (B) Heteromorphism of the tumor. (C) and (D) Juncture of the tumor.
Clinically, hepatic SFTs cause symptoms only after reaching a certain size, or when vital structures are involved. SFTs are not known to grow invasively, and the course of the disease following complete surgical resection is usually benign. The patient may be asymptomatic or have non-specific clinical manifestations, including increased abdominal volume and circumference and abdominal pain, distention, and discomfort, as seen in our patient, or other symptoms, such as anorexia, nausea, vomiting and weight loss. Fever, hypoglycemia, abnormal liver tests and biliary-duct compression that leads to cholestasis and progressive jaundice are less commonly seen $[12,13,16,19]$. Laboratory results are usually normal; however, a few patients have had deranged liver function tests or elevated serum levels of a-fetoprotein $[15,19,20]$. Our patient had a high level of serum CA125.

The radiological findings of SFTs are unspecific and cannot distinguish between benign and malignant tumors. The diagnosis should be suspected if imaging reveals a solitary, highly vascular, well circumscribed, encapsulated mass that shows heterogeneous enhancement (due to the varied density of the collagen component) in CT and MRI images, especially if the enhancement is hypointense on the T2-weighted MRI and shows progressive enhancement in the delayed phase [20]. Most benign or malignant liver tumors, including hemangiomas, hepatic adenomas, focal nodular hyperplasia, hepatocellular carcinomas, fibrolamellar hepatocellular carcinomas and peripheral mass-forming cholangiocarcinomas, are predominantly hyperintense on the T2-weighted images, and some have a delayed, persistent enhancement [21]. The imaging features of benign and malignant solitary fibrous liver tumors appear to overlap. DWI can differentiate solitary fibrous tumors from malignant liver tumors. A whole-body fluorodeoxyglucose positron emission tomography (PET) showed that the mass had low metabolic activity and was probably benign $[13,22]$.

Several authors believe that fine-needle aspiration also helps to establish a diagnosis, if the tissue sample is adequate and the fine-needle aspiration can be analyzed for immunomarkers; there has been one successful case report of this practice [23]. Other authors regard fineneedle biopsy to be inaccurate and do not recommend this practice. Large solid lesions of the liver (irrespective of the diagnosis), if deemed resectable at the pre- and intraoperative assessment, should be excised and sent for histological examination. Specimens obtained from a fine-needle biopsy only represent a small area of the tumor and may be misleading when entrapped proliferating bile ducts are mistaken for an adenocarcinoma, as in the present case. Alternatively, malignant foci may be missed, which would give a falsely reassuring result. 

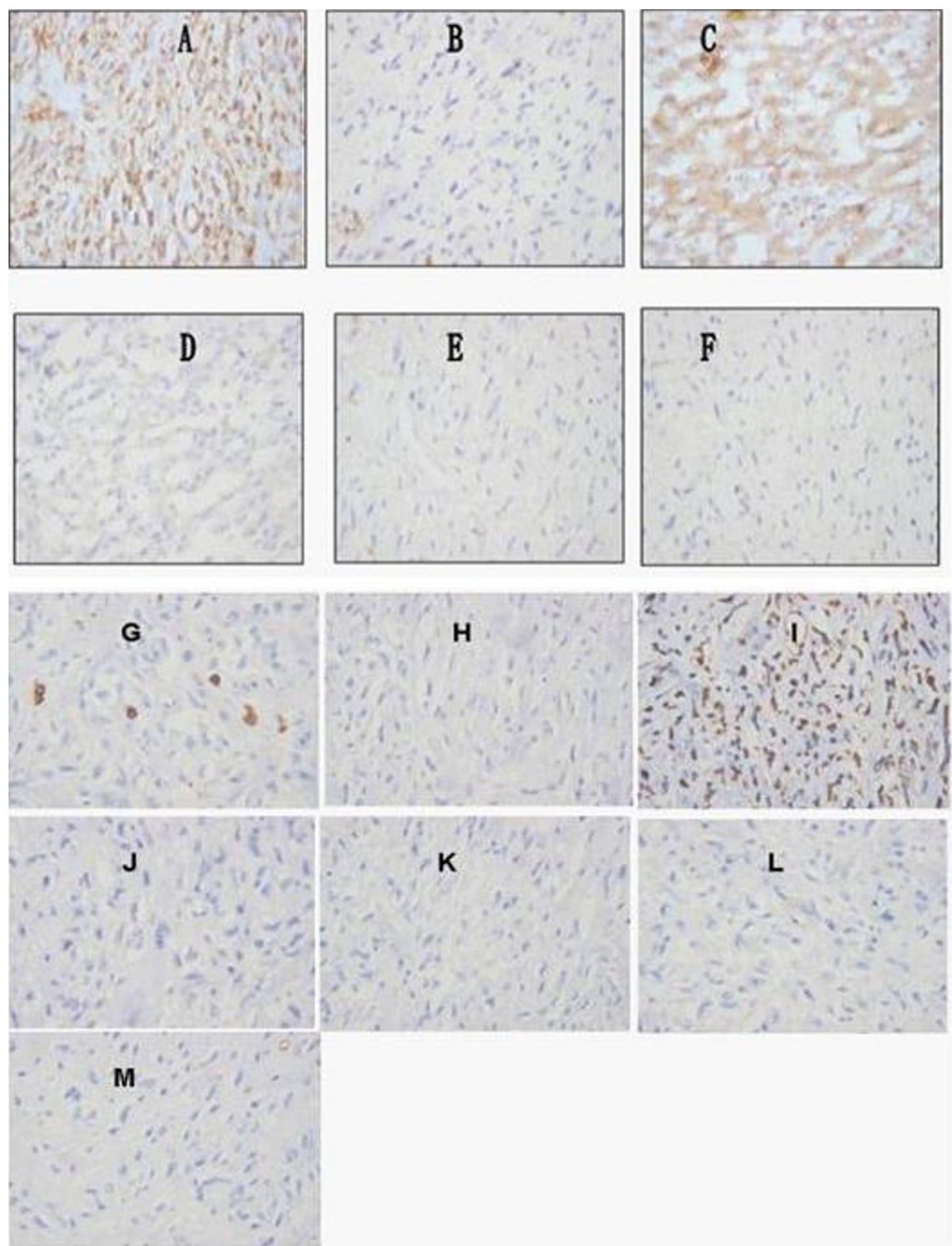

Figure 6 Immunohistochemical features. (A) Diffuse and strong CD34 immunopositivity. (B) Negative CD68 antigen staining. (C) Positive bcl-2 antigen staining. (D) Negative CD99 antigen staining. (E). Diffusely negative S-100 staining. (F) Negative smooth-muscle actin.(G) 3-5\% positive Ki67 antigen staining. (H) Negative cytokeratin antigen staining. (I) Positive vimentin antigen staining. (J) Negative synaptophysin antigen staining. (K) Negative calretinin. (L) Negative Desmin antigen staining. (M) Negative CD31 antigen staining. 


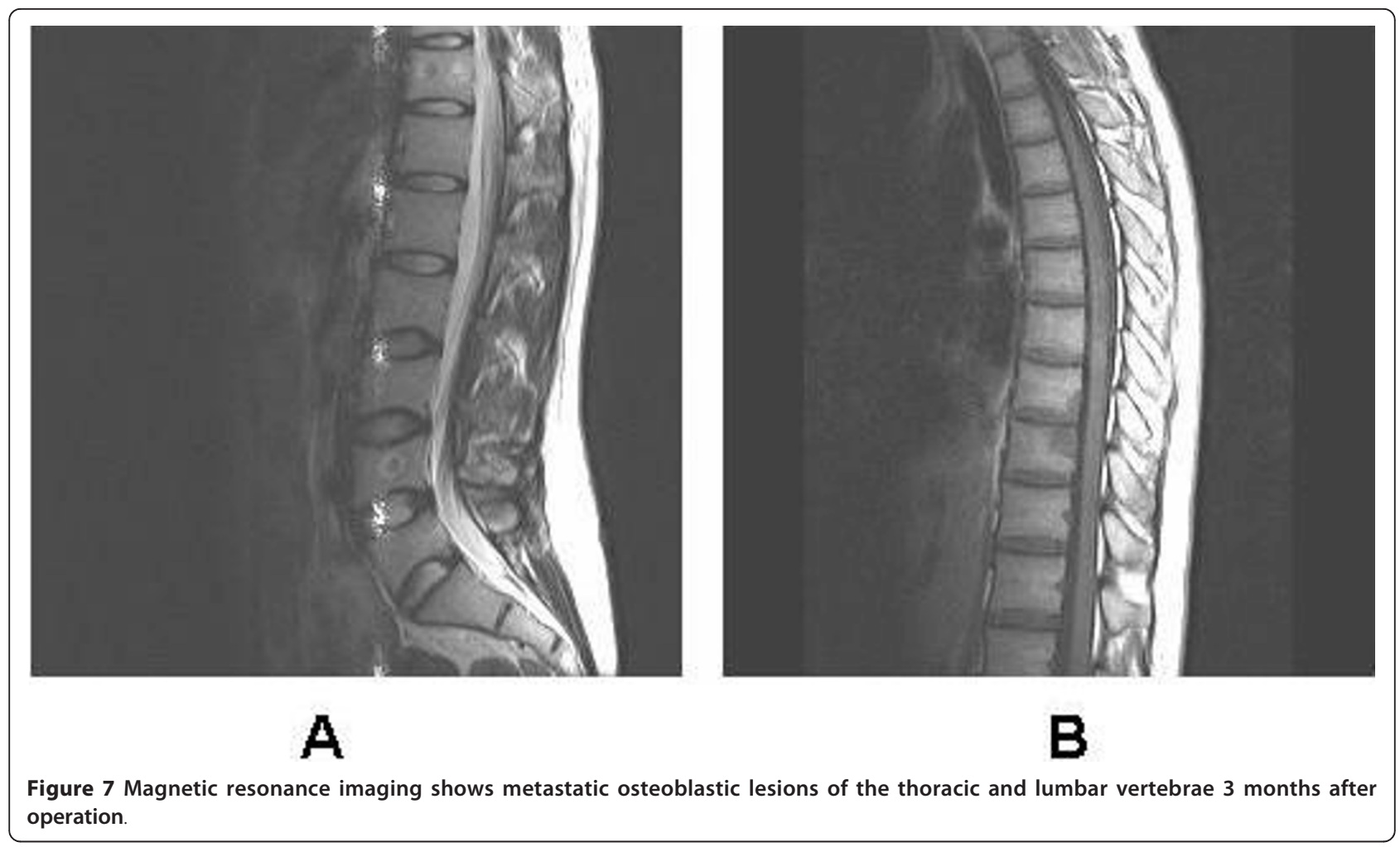

Moreover, if the tumor is malignant, a percutaneous biopsy may seed tumor cells along the biopsy tract $[7,22]$.

The tumor may be found on either the right or left side of the liver. The average tumor weight, where available, was $3,184 \mathrm{~g}$ and ranged from $1,850 \mathrm{~g}$ to $4,500 \mathrm{~g}$. The mean of the largest diameter was $17.8 \mathrm{~cm}(2-32$ $\mathrm{cm}$ )[18]. Paula Novais [24] presented a case of a large solitary hepatic fibrous tumor that was unresectable at first. Two years later, another attempt at surgical resction was made, but a life-threatening surgical complication occurred during the postoperative course. Unresectable, rare, non-hepatocellular or bile duct tumors that arise within the hepatic parenchyma are a formal indication for liver transplantation; therefore, liver transplantation can be considered for non-resectable SFTs. Because the patient was stable for 4 years, the author questioned such aggressive treatments in asymptomatic patients, due to the natural history of this tumor, which is not well known, and the risk of other complications.

In the present case, the liver SFT diagnosis was based on the association of characteristic histological and immunohistochemical features (i.e., high cellular proliferation of spindle cells arranged in a storiform pattern) with the immunohistochemical staining profile of CD34 $(+), \mathrm{Bcl}-2(+)$ and SM-actin (-). These characteristics differentiate SFTs from other liver tumors, such as primary

Table 1 Clinicopathological features of the reported malignant cases of liver solitary fibrous tumors

\begin{tabular}{|c|c|c|c|c|c|c|c|c|c|}
\hline No & Reference & Year & $\begin{array}{l}\text { Age/ } \\
\text { sex }\end{array}$ & Treatment & Size $(\mathrm{cm})$ & $\begin{array}{l}\text { Lobe } \\
\text { of } \\
\text { liver }\end{array}$ & Immunohistochemistry & Histology & $\begin{array}{l}\text { Follow- } \\
\text { up }\end{array}$ \\
\hline 1 & $\begin{array}{l}\text { Sezal } Y_{1} \\
\text { et al. [12] }\end{array}$ & 2000 & $25 / F$ & Excision & $32 \times 30 \times 12$ & Right & Vimentin+ & spindle cells with oval-fusiform nuclei & $\begin{array}{l}6 \\
\text { months }\end{array}$ \\
\hline 2 & $\begin{array}{l}\text { Chan G, } \\
\text { et al. [13] }\end{array}$ & 2007 & $70 / \mathrm{M}$ & Excision & $27 \times 24 \times 12$ & Right & $\begin{array}{l}\text { Vimentin+, } \\
\text { CD34+, Bcl2+, CD99+ }\end{array}$ & $\begin{array}{l}\text { spindle-cell fibrosarcoma: highly cellular and } \\
\text { pleomorphic, with areas of necrosis and a } \\
\text { very high mitotic index (> 20/hpf) }\end{array}$ & $\begin{array}{l}>9 \\
\text { months }\end{array}$ \\
\hline 3 & $\begin{array}{l}\text { Brochard } \\
\text { C, et al. } \\
{[14]}\end{array}$ & 2010 & $54 / \mathrm{M}$ & Excision & $17 \times 12 \times 8$ & Right & $\begin{array}{l}\text { Vimentin+, CD34+, } \\
\text { desmint, actin+ }\end{array}$ & $\begin{array}{l}\text { Tumor cells were polymorphic: small and } \\
\text { round, spindle-shaped, voluminous with } \\
\text { acidophilic cytoplasm, or with polymorphous } \\
\text { nuclei. }\end{array}$ & 9 years \\
\hline
\end{tabular}


hepatocellular carcinomas (CD34-negative), leiomyomas (smooth-muscle actin-positive and CD34-negative) and mesotheliomas (vimentin-positive, CD34-positive, cytokeratin-positive) [25]. However, the CD34 antibody is not specific for SFT diagnosis and can be positive in angiosarcomas and gastrointestinal stromal tumors.

A hepatic SFT is often a benign neoplasm that has a good prognosis after surgery without recurrence. Most authors believe that postoperative adjuvant chemotherapy or radiotherapy should be reserved for incomplete resections and/or pathological features of malignancy $[25,26]$. Currently, the combination of doxorubicin and ifosfamide is the standard systemic chemotherapy regimen for many subtypes of soft-tissue sarcomas. Gemcitabine with docetaxel has also emerged as a good therapeutic choice for these patients. Although cases of SFT that respond to these chemotherapeutic agents have been reported sporadically, no systematic review or clinical trial has identified an effective systemic regimen for an unresectable SFT to date[11]. However, it has been proven that standard chemotherapy regimens may only have limited efficacy in SFT. The combination of temozolomide and bevacizumab had a remarkably high rate of overall response and a favorable duration of disease control; temozolomide/bevacizumab is a promising therapeutic regimen that warrants further investigation.

When complete resection is impossible or when there is recurrence, radiotherapy can be used, but a negative surgical margin does not mean that radiotherapy is unnecessary. Postoperative radiotherapy may theoretically reduce the risk of local recurrence; therefore, postoperative radiotherapy should be used as an adjunctive therapy in all patients [27]. In our case, we also used TACE for arterial embolization several days before operation. TACE is an option to generate tumor hypotrophy for a better resection and reduced hemorrhagic risk. Almost all reports agree that a complete surgical resection with tumor-free margins remains the cornerstone of treatment because of the reported 10-year overall survival rates of 54-89\% [11]. The current case had widespread skeletal metastases; therefore, we used chemotherapy (adriamycin and ifosfamide), but this systemic regimen seemed to be inadequate. We believe that this report will contribute to understanding the natural history of liver SFTs.

SFTs of the liver are a rare neoplasm that cannot be definitely diagnosed by characteristic histological and immunohistochemical properties. A complete surgical resection with tumor-free margins is the best choice for treatment. Postoperative adjuvant chemotherapy or radiotherapy should be used in malignant SFTs; however, the use of these therapies in benign SFTs is still controversial.

\section{Consent}

Written informed consent was obtained from the patient for publication of this case report and accompanying images. A copy of the written consent is available for review by the Editor-in-Chief of this journal.

\section{Authors' contributions}

QL participated in the design of the study, and drafted the final version of the manuscript. LP participated in design of the study and manuscript drafting, carried out histopathological evaluation. YL participated in histopathological evaluation and helped in drafting the manuscript. YA tracked the clinical data. ZL helped in drafting the manuscript. YH tracked the clinical data. All authors read and approved the final manuscript.

\section{Competing interests}

The authors declare that they have no competing interests.

Received: 20 October 2011 Accepted: 22 December 2011 Published: 22 December 2011

\section{References}

1. Klemperer P, Rabin CB: Primary neoplasm of the pleura: a report of five cases. Arch Pathol Lab Med 1931, 11:385-412.

2. Guillou L, Fletcher JA, Fletcher CDM, Mandahl N: Extrapleural solitary fibrous tumor and hemangiopericytoma. In Pathology and Genetics of Tumours of Soft Tissues and Bone. Edited by: Fletcher CDM, Unni KK, Mertens F. Lyon, France: IARC Press; 2002:86.

3. Churg A, Cagle PT, Roggli VL: Solitary fibrous tumor. Tumors of the Serosal Membranes. 4 edition. Washington, DC: American Registry of Pathology Press; 2006, 299

4. Chan JK: Solitary fibrous tumour-everywhere, and a diagnosis in vogue. Histopathology 1997, 31:568-576.

5. Ji Y, Fan J, Xu Y, Zhou J, Zeng HY, Tan YS: Solitary fibrous tumor of the liver. Hepatobiliary Pancreat Dis Int 2006, 5:151-153

6. Filosso PL, Asioli S, Ruffini E: Radical resection of a giant, invasive and symptomatic malignant Solitary Fibrous Tumour (SFT) of the pleura. Lung Cancer 2009, 64:117-120.

7. Bishop JA, Rekhtman N: Malignant Solitary Fibrous Tumor Cytopathologic Findings and Differential Diagnosis. Cancer (Cancer Cytopathol) 2010, 118(2):83-9.

8. Dalton WT, Zolliker AS, McCaughey WT, Jacques J, Kannerstein M: Localized primary tumors of the pleura: an analysis of 40 cases. Cancer 1979, 44:1465-75.

9. England DM, Hochholzer L, McCarthy MJ: Localized benign and malignant fibrous tumors of the pleura. A clinicopathologic review of 223 cases. Am J Surg Pathol 1989, 13:640-58

10. Witkin GB, Rosai J: Solitary fibrous tumor of the mediastinum. A report of 14 cases. Am J Surg Pathol 1989, 13:547-57.

11. Park M, Patel S, Ludwig J, Trent J, Conrad C, Lazar A, Wang W, Boonsirikamchai P, Choi H, Wang X, Benjamin R, Araujo D: Activity of Temozolomide and Bevacizumab in the Treatment of Locally Advanced, Recurrent, and Metastatic Hemangiopericytoma and Malignant Solitary Fibrous Tumor. Cancer 2011.

12. Yilmaz S, Kirimlioglu V, Ertas E, Hilmioglu F, Yildirim B, Katz D, Mizrak B: Giant Solitary Fibrous Tumor of the Liver with Metastasis to the Skeletal System Successfully Treated with Trisegmentectomy. Digestive Diseases and Sciences 2000, 45:168-174.

13. Chan G, Horton P, Thyssen S, Lamarche M, Nahal A, Hill D, Marliss E, Metrakos P: Malignant transformation of a solitary fibrous tumor of the liver and intractable hypoglycemia. J Hepatobiliary Pancreat Surg 2007, 14:595-9.

14. Brochard C, Michalak S, Aubé C, Singeorzan C, Fournier HD, Laccourreye L, Calès $P$, Boursier $\mathrm{J}$ : A not so solitary fibrous tumor of the liver. Gastroentérologie Clinique et Biologique 2010, 34:716-720.

15. Moran C, Ishak K, Goodman Z: Solitary fibrous tumor of the liver: a clinicopathologic and immunohistochemical study of nine cases. Ann Diagn Pathol 1998, 2:19-24 
16. Nakamura S, Fukui M, Takeshita H, Tsuji Y, Murata H, Kubo T: Malignant solitary fibrous tumor of the thigh accompanied by hypoglycemic coma. A case report. Anticancer Res 2005, 25:2461-4.

17. Jia C, Shi S, Zhao Z, Zhen S: Solitary fibrous tumor of the liver: retrospective study of reported cases. Cancer Invest 2006, 24:132-5.

18. Neeff $H$, Obermaier $R$, Technau-Ihling $K$, Werner M, Kurtz $C$, Imdahl $A$, Hopt UT: Solitary fibrous tumour of the liver: case report and review of the literature. Langenbecks Arch Surg 2004, 389:293-298.

19. Fuksbrumer $M$, Klimstra D, Panicek D: Solitary fibrous tumor of the liver: imaging findings. AJR Am J Roentgenol 2000, 175:1683-1687.

20. Guglielmi A, Frameglia M, Iuzzolino P, Martignoni G, DeManzoni G, Laterzi E, Veraldi G, Girlanda R: Solitary fibrous tumor of the liver with CD 34 positivity and hypoglycemia. J Hepatobiliary Pancreat Surg 1998, 5:212-6.

21. Kandpal H, Sharma R, Gupta SD, Kumar A: Solitary fibrous tumour of the liver: a rare imaging diagnosis using MRI and diffusion-weighted imaging. The British Journal of Radiology 2008, 81:e282-e286.

22. Chen J, Ong S, Richards C, Garcea G, Pollard C, Berry D, Dennison A: Inaccuracy of Fine-needle Biopsy in the Diagnosis of Solitary Fibrous Tumour of the Liver. Asian J Surg 2008, 31(4):195-8.

23. Clayton A, Salomao D, Keeney G, Nascimento A: Solitary fibrous tumor: a study of cytologic features of six cases diagnosed by fine-needle aspiration. Diagn Cytopathol 2001, 25:172-6.

24. Novais P, Robles-Medranda C, Pannain VL, Barbosa D, Biccas B, Fogaça H: Solitary Fibrous Liver Tumor: Is Surgical Approach the Best Option? March 2010, 19(1):81-84.

25. Korkolis D, Apostolaki K, Aggeli C, Plataniotis G, Gontikakis E, Volanaki D, Sebastiadou M, Dimitroulopoulos D, Xinopoulos D, Zografos G, Vassilopoulos P: Solitary fibrous tumor of the liver expressing CD34 and vimentin: A case report. World I Gastroenterol 2008, 14(40):6261-6264.

26. Archontaki M, Korkolis D, Arnogiannaki N, Hatzijiannis $S$, Dendrinos $P$, Megapanos C, Kassotakis D, Kokkalis G: Histologically Malignant Solitary Fibrous Tumour of the Anterior Thoracic Wall: A Case Report and Review of the Literature. Case Reports in Medicine 2010, 257167-257171.

27. Yang $X$, Zheng J, Ye W, Wang Y, Zhu H, Wang L, Zhang Z: Malignant solitary fibrous tumors of the head and neck: A clinicopathological study of nine consecutive patients. Oral Oncology 2009, 45:678-682.

doi:10.1186/1746-1596-6-127

Cite this article as: Peng et al: Skull base metastases from a malignant solitary fibrous tumor of the liver. A case report and literature review. Diagnostic Pathology 2011 6:127.

\section{Submit your next manuscript to BioMed Central and take full advantage of:}

- Convenient online submission

- Thorough peer review

- No space constraints or color figure charges

- Immediate publication on acceptance

- Inclusion in PubMed, CAS, Scopus and Google Scholar

- Research which is freely available for redistribution

Submit your manuscript at www.biomedcentral.com/submit 